\title{
Image Authentication Scheme Based on Self-embedding Watermarking
}

\author{
Clara Cruz-Ramos, Rogelio Reyes-Reyes, Mariko Nakano-Miyatake, \\ and Héctor Pérez-Meana \\ ESIME Culhuacan, National Polytechnic Institute of México \\ Av. Santa Ana No. 1000, Col. San Francisco Culhuacan, México D.F., México \\ ccruza@ipn.mx, mariko@infinitum.com.mx
}

\begin{abstract}
This paper presents a block-wise and content-based semi-fragile image watermarking authentication scheme with location and recovery capability. Firstly the image is segmented by two regions: Region of Interest (ROI) and Region of Embedding (ROE). The watermark sequence is extracted from ROI and it is embedded into the middle frequency band of DCT coefficients of ROE. In the authentication stage, two watermark sequences extracted ROI and ROE, respectively, are used. If difference between both sequences of a ROI block is smaller than the predefined threshold value, the ROI block is determined authentic, otherwise the block is considered as tampered and it is recovered by the recovery process. The proposed scheme is evaluated from several points of view: watermark imperceptibility, capability of tamper detection, image quality of recovered regions and robustness of no-intentional manipulations, such as JPEG compression. The simulation results show fairly good performance of the proposed scheme.
\end{abstract}

Keywords: Image authentication, Semi-fragile watermarking, Self-embedding watermarking, Tamper detection, Tamper localization.

\section{Introduction}

With the growth of Internet, digital images play an important role to show some evidences in the news and reports in the digital media. Digital images captured by remote sensing technique provide us important information in several fields. However, using some software tools, digital images can be modified easily without any traces, causing economic and social damages. Therefore development of a reliable digital image authentication scheme is an urgent issue. Among several approaches, a watermarking based approach is considered as one of alternative solutions. In general, image authentication schemes can be classified into two approaches: signature-based authenticators [1,2] and watermarking-based authenticators [3-6]. The major difference between these two approaches is that the authentication message is embedded into the same digital media in watermarking-based authenticators, while it is transmitted or saved separately from digital media in the signature-based authenticators. In watermarking-based authenticators, they can be further classified into two schemes: fragile (complete authentication) [3, 4] or semi-fragile (content authentication) [5, 6] watermarking authentication schemes. 
Many watermarking based methods determine if the image is tampered or not, and some of them can localize the tampered regions [3-6], however, very few schemes have capability to recover the tampered region without original image $[7,8]$. In this paper, an image authentication scheme, with a capability of tampered region localization and recovery, is proposed. In the proposed scheme, an image is segmented by two regions: Regions of Interest (ROI) and Regions of Embedding (ROE). ROI is a region which contains important information and it is required protection, for example regions of faces of persons involved in some scandal scene, while ROE is rest of the whole image after subtracting region belonged to ROI. ROE can be background of the image. The information of ROI is encoded to generate watermark sequence, and it is embedded into ROE of the same image in an imperceptible manner. In the authentication stage, two watermark sequences, extracted from ROI and ROE respectively, are used. If some blocks of ROI are detected as tampered, the recovery process performs to construct these blocks from the watermarked sequence extracted from ROE.

The proposed scheme is evaluated from several points of view: watermark imperceptibility in the watermarked image, capability of tampered regions detection, recovery capability of the altered region, and watermark robustness against no-intentional modification, such as JPEG compression. Simulation results show a fairy good performance about above three issues.

The rest of the paper is organized as follows. In Section 2, the proposed authentication method is described, and in Section 3 the experimental results are provided. Finally a conclusion of this paper is described in Section 4.

\section{The Proposed Authentication Method}

\subsection{Watermark Sequence Generation}

Generally in a photo image, some objects or some regions contain information more important than other regions. For example in an image of traffic accident, perhaps the regions of license plates of vehicles involved with the accident are more important than its background or other vehicles no related with the event. Therefore we define two regions in the image: region of interest (ROI) and region of embedding (ROE).

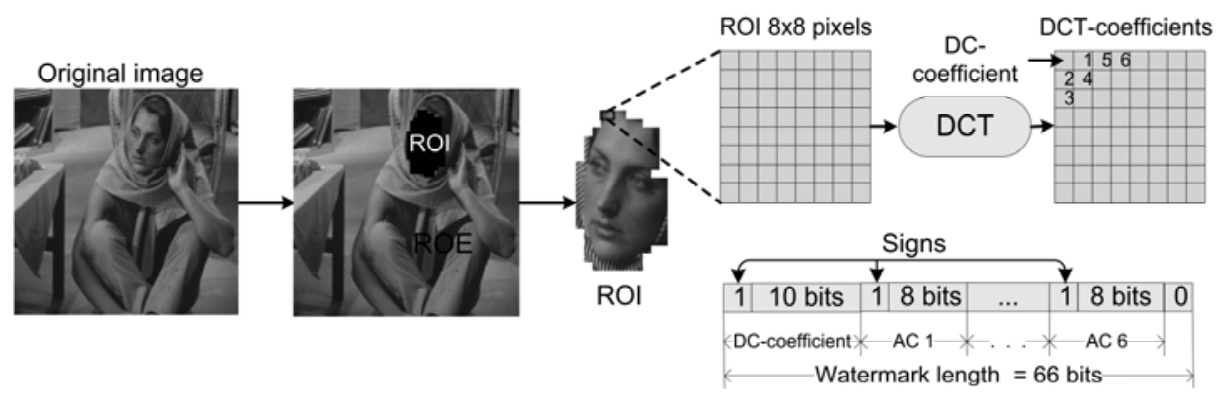

Fig. 1. Watermark sequence generation stage 
ROI is important region of the image that requires a protection against malicious modification, while ROE is the rest of the image that no requires any protection. In the proposed algorithm, information of ROI is extracted to generate a watermark sequence and this sequence is embedded into ROE. Figure 1 illustrates the proposed watermark generation process that can be summarized as follows:

1) Subtract 127 from gray levels of the original image to force pixel values to be $[-127,128]$. It reduces DC-coefficient value after the image is transformed by DCT.

2) In the original image $X$, ROI is selected by owner and automatically ROE is determined in order that the following condition is satisfied.

$$
R O I \cap R O E=\emptyset \text { and } R O I \cup R O E .
$$

3) ROI region is divided into non-overlapping blocks of $8 \times 8$ pixels.

4) In each block of ROI, 66 bits watermark sequence is extracted as a following manner.

a) Compute the 2D-DCT.

b) The DC-coefficient is rounded and represented by 11 bits (10 bits for absolute value and 1 bit for sign). Because the maximum values of DC for $8 \times 8$ block of an image with range $[-127,128]$ is 1016 , it can be represented in a binary form using 11 bits, including sign bit.

c) Encode each one of the first 6 lowest AC-coefficients, taking first $6 \mathrm{AC}$ coefficients in the zig-zag order of the block, to 8 bits together with 1 sign bit (total 9 bits).

5) The length of watermark sequence of each ROI block is 66 bits, composed by 11 bits of DC-coefficient, 54 bits corresponded to the 6 AC-coefficients of DCT coefficients and finally we add 1 zero, which can be divided into 6 segments with 11 bits sequence per segment.

\subsection{Watermark Embedding}

The proposed watermark embedding process can be summarized as follows:

1) Using a user's key K, the mapping list between ROI blocks and ROE blocks is constructed.

2) Using this mapping list, each ROI block of $8 \times 8$ pixels is mapped into $6 \mathrm{ROE}$ blocks, which are used to embed watermark sequence extracted from the ROI block.

3) In each selected $6 \mathrm{ROE}$ blocks, following processes are carried out.

a) Apply 2D-DCT to 6 ROE blocks.

b) Quantify by a quantized matrix Q that corresponds to quality factor 70 . This value is selected considering tradeoff between watermarked image quality and watermark robustness against JPEG compression. Quantization of DCT coefficients by Q is given by (2). 


$$
\tilde{C}(u, v)=\lfloor C(u, v) / Q(u, v)\rfloor .
$$

Where $\mathrm{C}(\mathrm{u}, \mathrm{v})$ and $\tilde{\mathrm{C}}(\mathrm{u}, \mathrm{v})$ are the $(\mathrm{u}, \mathrm{v})$-th DCT coefficient and it quantized version, respectively, $[\mathrm{x}]$ is lower nearest integer value of $\mathrm{x}$.

c) Each 11 bits of watermark sequence is embedded into the LSB of the 11 DCT-coefficients of the middle frequency band of the selected $6 \mathrm{ROE}$ blocks.

d) The watermarked DCT blocks are multiplied by Q.

e) It is transformed by the inverse DCT to get watermarked blocks.

4) Concatenating all watermarked blocks, the watermarked image is generated.

\subsection{Authentication and Recovery}

The authentication procedure verifies if the contents of the received image are authentic or not. To authenticate the image, two watermarks must be extracted and then compared. This authentication and recovery process are described as follows:

1) The first watermark $W_{\text {ROIext }}$ is generated from the ROI blocks; these operations are same as the watermark generation process before described.

2) The second watermark $W_{\text {ROEext }}$ is extracted from the ROE blocks. Using the same secret key to construct ROI-ROE mapping lists, the 6 corresponded ROE blocks are determined for each ROI block, from which $\mathrm{W}_{\text {ROEext }}$ is extracted.

3) For selected $6 \mathrm{ROE}$ blocks, the following operations are carried out to get $\mathrm{W}_{\text {ROEext }}$

a) Apply 2D-DCT to each one of 6 ROE blocks.

b) DCT blocks are quantized by quantification matrix $Q$.

c) 11 bits sequence is extracted from LSB of $11 \mathrm{AC}$ coefficients in the middle frequency band of each ROE block.

d) Concatenated 6 extracted sequences of longitude 11 bits to generate 66 bits $\mathrm{W}_{\text {ROEext }}$.

4) In the watermark comparison between $W_{\text {ROIext }}$ and $W_{\text {ROEext }}$, the tolerant threshold $T h$ is employed to distinguish a content preserving operation from malicious manipulation. This authenticity check is given by (3).

$$
\begin{aligned}
& \text { if } \sum X O R\left(W_{\text {ROIext }}, W_{\text {ROEext }}\right)<T h \text { then the block is authentic } \\
& \text { if } \sum X O R\left(W_{\text {ROIext }}, W_{\text {ROEext }}\right) \geq T h \text { then the block is modified }
\end{aligned}
$$

Once the authenticity check indicates that a ROI block was tampered, the recovery process of this ROI block is triggered. The recovery process can be summarized as follows:

1) From the extracted watermark sequence $W_{\text {ROEext }}$, last bit is eliminated to get a watermark sequence with 65 bits.

2) Assign the first 11 bits of $\mathrm{W}_{\text {ROEext }}$ to DC-component and the rest 54 bits are divided into 6 sequences with 9 bits and these are assigned to 6 lowest ACcoefficients of a recovery DCT block. 
3) Compute the inverse 2D-IDCT of recovery DCT block to get a recovery block.

4) Replace the tampered ROI block by the recovery block.

\section{Experimental Results}

We conduct three experiments to evaluate performance of the proposed algorithm. The first experiment is to assess watermark imperceptibility, and in the second one the tamper detection and the recovery capability of the proposed algorithm are evaluated. Finally, in the third experiment, the watermark robustness to incidental modification such as JPEG compression is evaluated. In table 1, the values of some factors used during the evaluation are given.

Table 1. Parameter's values used during the evaluation process

\begin{tabular}{|l|c|c|}
\hline Number of test images & $\begin{array}{c}256-\text { gray level } \\
(8 \text { bits/pixel })\end{array}$ & 100 \\
\hline Length of watermark sequence for each ROI block & $\mathrm{W}$ & 66 bits \\
\hline Threshold value used in (3) & Th & 13 \\
\hline Number of ROI blocks used & {$[$ min, max $]$} & {$[117,453]$} \\
\hline
\end{tabular}

Experimental results of three evaluations are described in the following sections.

\subsection{Watermark Imperceptibility}

Gray-scale images are used for these experiments. Figure 2 shows watermark imperceptibility using two images "Car" and "Camera" as examples. Figs. 2(a) and 2(d) show original image and figs. 2(b) and 2(e) show the watermarked images, respectively. Peak signal to noise ratio (PSNR) between the original image and the watermarked one are $36.8 \mathrm{~dB}$ and $33.17 \mathrm{~dB}$, respectively. These results indicate that the image distortions incurred by the watermark embedding process are not significant. Also, in perceptual comparison by human visual system between the original images and the watermarked one, it is difficult to distinguish the difference between both images.

The watermark length of the cover image depends directly on the number of ROI blocks selected by the owner, and also watermarked image distortion depends on the embedded watermarked length. Figs. 2(c) and 2(f) show an example of possible ROI blocks selected by the owner, which are represented by black squares. Here $4 \%$ of blocks in Fig. 2(c) and 14.8\% of blocks in Fig 2(f) are selected as ROI blocks. Fig. 3 shows a plot of the PSNR as a function with number of ROI blocks. In this figure, watermarked image quality (PSNR) is inversely proportioned by number of ROI blocks. The number of ROI blocks must satisfy (4), because each ROI block requires 6 ROE blocks.

$$
\operatorname{Number}(R O I)<N B(I) /_{7}
$$

where $N B(I)$ is total number of blocks ( $8 \times 8$ pixels) of the Image $\mathrm{I}$. 


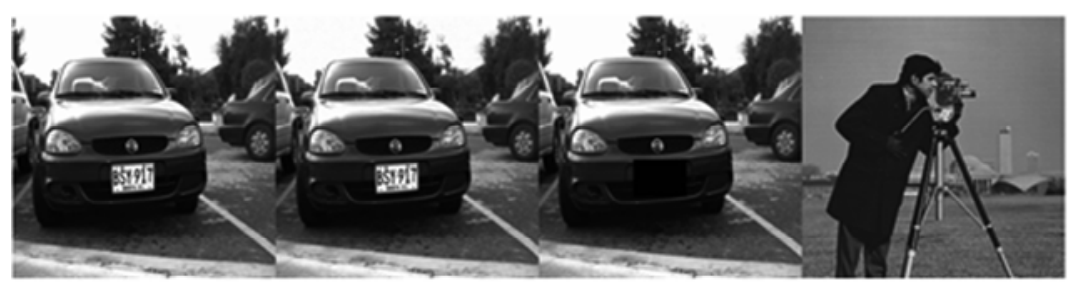

(a)

(b)

(c)

(d)

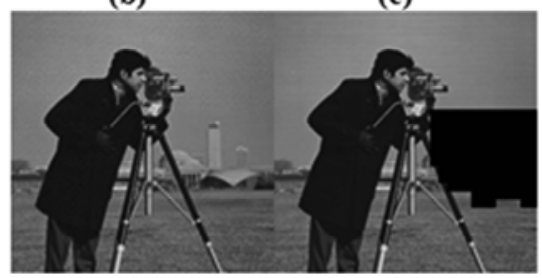

(e)

(f)

Fig. 2. Watermark imperceptibility, (a,d) Original images, (b,e) Watermarked images, (c,f) ROI blocks indicated by black blocks, which are assigned by owner of the image

\subsection{Tamper Detection and Recovery Capability}

To evaluate the tamper detection and recovery of the proposed authentication scheme, the watermarked images were tampered as shown by fig. 4(b) and (f). In the fig. 4 (b), number ' 7 ' of license plate is tampered, modifying ' 9 ' and in the fig. 4 (f), a tower behind cameraman is eliminated. As shown by fig. 2(c) and 2(f), tamped regions are assigned as ROI blocks. The tamper detection results are shown by fig. 4(c) and (g), where tampered ROI blocks are represented by black squares, and fig. 4(d) and (h) show images that the tampered regions were recovered.

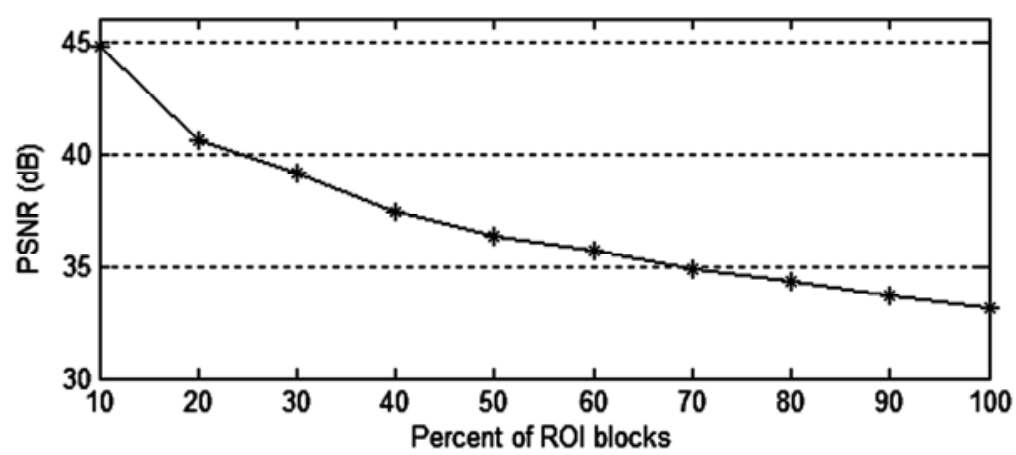

Fig. 3. Relationship between number of ROI blocks and watermarked image distortion

\subsection{Watermark Robustness}

Generally any images, including watermarked image, suffer some no-intentional modifications, such as compression or noise contamination, therefore, watermark robustness against these incidental modifications must be taken into account. In the 


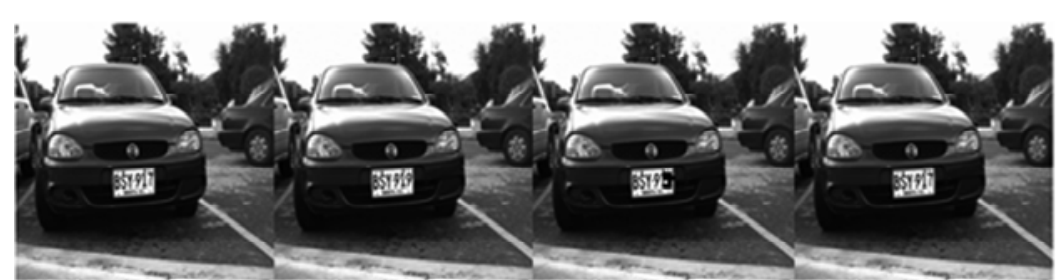

(a)

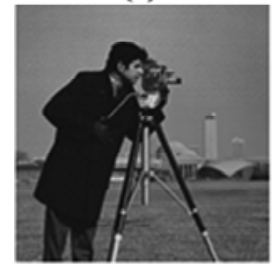

(e) (b)

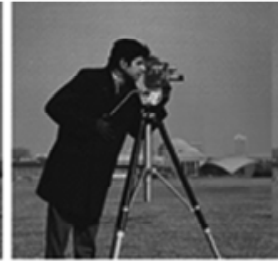

(f) (c)

(d)

Fig. 4. $(\mathrm{a}, \mathrm{e})$ are watermarked images, $(\mathrm{b}, \mathrm{f})$ are tampered images, $(\mathrm{c}, \mathrm{g})$ show detection of tampered regions and $(\mathrm{d}, \mathrm{h})$ are recovered images

proposed authentication method, ROI information is embedded as watermark sequence into quantized DCT coefficients, which is generated by a predefined JPEG quality factor. This embedding domain guarantees that watermark sequence can be extracted in almost intact manner, after watermarked image suffer JPEG compression with a better quality factor than the predefined one. Therefore in the proposed scheme, embedded watermark sequence is robust to JPEG compression with a quality factor better than 70 .

\section{Conclusions}

In this paper, a block-based image authentication with tamper detection and recovery capability is proposed. Firstly image is segmented by two regions: Regions of Interest (ROI) and Regions of Embedding (ROE). The watermark sequence is a compressed version of each ROI block and it is encoded a binary sequences with 66 bits. Then the 66 bits watermark sequence of each ROI block is embedded into corresponded 6 ROE blocks in its DCT domain. In the authentication stage, watermark sequence extracted from ROI blocks of the image under analysis is compared with the watermark sequences extracted from ROE blocks. If some ROI blocks are determined as tampered blocks, the recovery processes is triggered, in which, the tampered ROI blocks are recovered from the watermark sequence extracted from ROE blocks. Computer simulation results show fairly good performance of the proposed scheme, analyzing watermark imperceptibility, tamper detection and recovery capability and watermark robustness against no intentional attacks, such as JPEG compression. In the proposed scheme, recovered image of the tampered ROI blocks are sufficiently clear after watermarked image is compressed by JPEG compression with a reasonable quality factor (better than 70). 
Acknowledgments. We thank to the National Polytechnic Institute of México and National Council for Science and Technology (CONACYT) of México for the support provided during the realization of this research.

\section{References}

1. Lu, C.-S., Liao, H.-Y.: Structural Digital Signature for Image Authentication: An Incidental Distortion Resistant Scheme. IEEE Trans. Multimedia 5(2), 161-173 (2003)

2. Lou, D.-C., Ju., J.-L.: Fault Resilient and Compression Tolerant Digital Signature for Image authentication. IEEE Trans. Consumer Electron. 46(1), 31-39 (2000)

3. Maeno, K., Sun, Q., Chang, S.-F., Suto, M.: New Semi-Fragile Image Authentication Watermarking Techniques Using Random Bias and Nonuniform Quantization. IEEE Trans. Multimedia 8(1), 32-45 (2000)

4. Wong, P.-W., Memon, N.: Secret and Public Key Image Watermarking Schemes for Image Authentication and Ownership Verification. IEEE Trans. Image Processing 10(10), 1593 1601 (2001)

5. Lin, C.-Y., Chang, S.-F.: A Robust Image Authentication Method Distinguishing JPEG compression from Malicious Manipulation. IEEE Trans. Circuit Syst. Video Technol. 11(2), $153-168(2001)$

6. Lu, Z.-M., Xu, D.-G., Sun, S.-H.: Multipurpose Image Watermarking Algorithm Based on Multistage Vector Quantization. IEEE Trans. Image processing 14, 822-831 (2005)

7. Lin, P.-L., Huang, P.-W., Peng, A.-W.: A Fragile Watermarking Scheme for Image Authentication with Localization and Recovery. In: Proc. of the IEEE sixth Int. Symp. on Multimedia Software Engineering, pp. 146-153 (2004)

8. Tsai, P., Hu, Y.-C.: A Watermarking-Based Authentication with Malicious Detection and Recovery. In: Int. Conf. of Information, Communication and Signal Processing, pp. 865869 (2005) 